\title{
Chemical Composition, Antibacterial, Antibiofilm and Synergistic Properties of Essential Oils from Eucalyptus globulus LABILL. and Seven Mediterranean Aromatic Plants
}

\author{
Maria Vieira, ${ }^{a, 1}$ Lucinda J. Bessa, ${ }^{* a, b, 1}$ M. Rosário Martins, ${ }^{c}$ Sílvia Arantes, ${ }^{d}$ António P. S. Teixeira, ${ }^{e}$ \\ Ângelo Mendes, ${ }^{a}$ Paulo Martins da Costa, ${ }^{a, f}$ and Anabela D. F. Belo ${ }^{g}$ \\ aDepartamento de Produção Aquática, Instituto de Ciências Biomédicas Abel Salazar (ICBAS), Universidade do Porto, \\ PT-4050-343 Porto \\ bUCIBIO/REQUIMTE, Departamento de Química e Bioquímica, Faculdade de Ciências da Universidade do Porto, \\ PT-4169-007 Porto, e-mail: lucinda.bessa@fc.up.pt \\ 'Laboratório HERCULES \& Departamento de Química, Escola de Ciências e Tecnologia, Universidade de Évora, \\ PT-7000-671 Évora
}

dICAAM - Instituto de Ciências Agrárias e Ambientais Mediterrânicas \& Laboratório HERCULES, Universidade de Évora, PT-7002-554 Évora

eCentro de Química de Évora \& Departamento de Química, Escola de Ciências e Tecnologia, Universidade de Évora, PT-7000-671 Évora

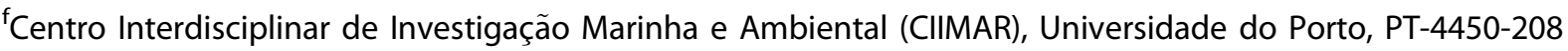
Matosinhos

IICAAM - Instituto de Ciências Agrárias e Ambientais Mediterrânicas, Departamento de Biologia, Escola de Ciências e Tecnologia, Universidade de Évora, PT-7002-554 Évora

Essential oils (EOs) from Eucalyptus globulus LABILL. ssp. globulus and from Mediterranean autochthonous aromatic plants - Thymus mastichina L., Mentha pulegium L., Rosmarinus officinalis L., Calamintha nepeta (L.) SAvI Ssp. nepeta, Cistus ladanifer L., Foeniculum vulgare L., Dittrichia viscosa (L.) GREUTER ssp. viscosa - were extracted by hydrodistillation and characterized by GC-FID and NMR spectroscopy. EOs were evaluated for antimicrobial properties against several bacterial strains, using diverse methods, namely, the agar disc-diffusion method, the microdilution method, the crystal violet assay and the Live/Dead staining for assessment of biofilm formation. Potential synergy was assessed by a checkerboard method. EOs of $R$. officinalis and $C$. ladanifer showed a predominance in monoterpene hydrocarbons (> 60\%); EOs of C. nepeta, M. pulegium, T. mastichina, E. globulus and F. vulgare were rich in oxygenated monoterpenes (62-96\%) whereas EO of D. viscosa was mainly composed of oxygenated sesquiterpenes (54\%). All EOs showed antimicrobial activity; $M$. pulegium and $E$. globulus generally had the strongest antimicrobial activity. EO of $C$. nepeta was the most promising in hampering the biofilm formation. The combinations D. viscosa/C. nepeta and E. globulus/ T. mastichina were synergistic against Staphylococcus aureus. These results support the notion that EOs from the aromatic plants herein reported should be further explored as potential pharmaceuticals and/or food preservatives.

Keywords: Wild-grown aromatic plants, Essential oils, Antibacterial activity, Antibiofilm, Synergistic effects.

\section{Introduction}

Recent years have seen an increased interest in the bioactive compounds produced by organisms such as plants, fungi and bacteria, since they are very likely to possess pharmacological and biotechnological potential. Bacteria resistant to common antibiotics have

1 These authors contributed equally to this work. reached alarming levels in many parts of the world indicating that many of the available treatment options for ordinary infections are becoming ineffective, which is causing prolonged illness, longer stays in hospitals and increased mortality. ${ }^{[1]}$ It is of consensus that the development of new antimicrobial drugs and alternative therapies is crucial and urgent.

Medicinal plants are an important source of research for the pharmaceutical and food industries. ${ }^{[2]}$ Particularly, in the past few decades, medicinal and 